\title{
H- and K- Band Methane Features in an L Dwarf, 2MASS $0920+35$
}

\author{
Tadashi Nakajima \\ National Astronomical Observatory, 2-21-1 Osawa, Mitaka, Tokyo, \\ 181-8588, Japan \\ Takashi Tsuji \\ Institute of Astronomy, University of Tokyo, 2-21-1 Osawa, Mitaka, \\ Tokyo, 181-0015, Japan \\ Kenshi Yanagisawa \\ Okayama Astrophysical Observatory, Kamogata, Okayama, 719-0232, \\ Japan
}

\begin{abstract}
We have obtained near-infrared spectra of three L dwarfs discovered by 2MASS, 1146+22 (L3), 1507-16 (L5), and 0920+35 (L6.5). From the comparison of the $\mathrm{H}$ and $\mathrm{K}$ band spectra of these $\mathrm{L}$ dwarfs, we have found the presence of methane absorption in $0920+35$. This implies that detectable methane absorption in the $\mathrm{H}$ and $\mathrm{K}$ bands, usually considered the signature of a $\mathrm{T}$ dwarf, can be present in objects classified optically as late L. Methane detection in L dwarfs is consistent with the presence of a dust layer deep in the atmosphere as the unified model of Tsuji suggests. (This work has been published in Nakajima, Tsuji \& Yanagisawa (2001).)
\end{abstract}

\section{Introduction}

From the point of view of observations, the sequence from $\mathrm{L}$ to early $\mathrm{T}$ dwarfs appears natural, but there are issues related to the $\mathrm{L} / \mathrm{T}$ transition that need to be resolved before we fully understand ultracool dwarfs.

We need a theoretical explanation of the $\mathrm{L} / \mathrm{T}$ transition process in which the behavior of colors and spectra must be understood. Models that take into account the effects of dust clouds have been proposed to explain the sequence from $\mathrm{L}$ to $\mathrm{T}$ and we wish to examine their validity (Marley et al. 2002; Tsuji 2002)

Another issue is the presence of methane in L dwarfs. Noll et al. (2000) confirmed the presence of methane by L band spectroscopy of L5V and L8V. Geballe et al. (2002) find that according to their classification scheme $\mathrm{K}$ band methane appears at $\mathrm{L} 8$ while $\mathrm{H}$ band methane marks the onset of the spectral class $\mathrm{T}$. It is worth revisiting the features in late $\mathrm{L}$ dwarfs taking into account the possible presence of methane absorption. 

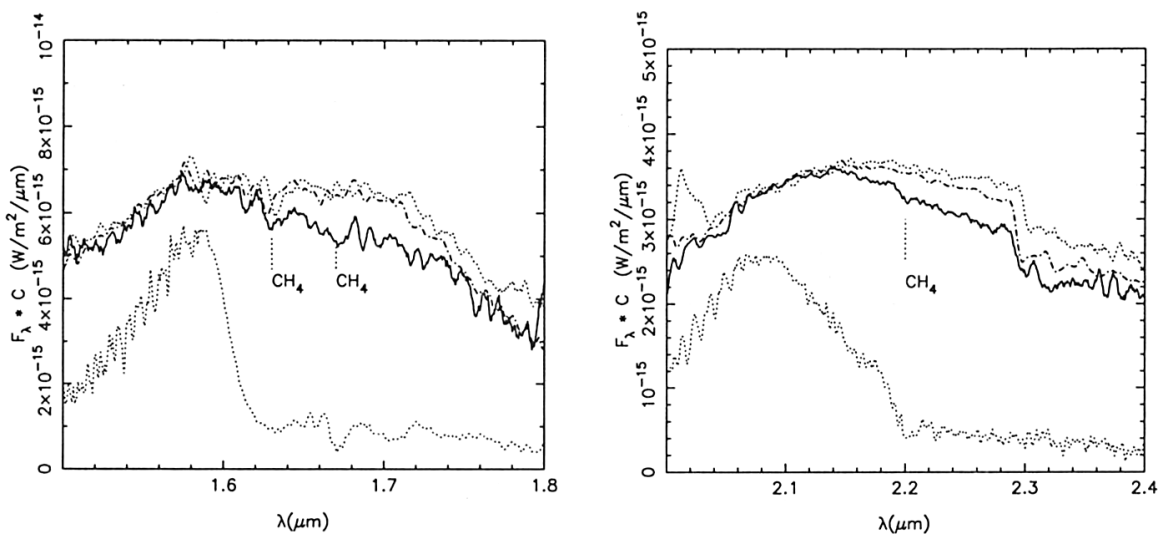

Figure 1. In Figures 1a and $1 \mathrm{~b}, \mathrm{H}$ and $\mathrm{K}$ band spectra are normalized so that portions of the spectra which are least affected by molecular absorption features overlap. To emphasize the locations of $\mathrm{H}_{2} \mathrm{O}$ and $\mathrm{CH}_{4}$ absorption features, the spectra of Gl 229B (Geballe et al. 1996) are also shown. In Figure 1a, flux depression of 2M0920+35 (solid) is conspicuous compared to the other two objects and the absorption maxima at 1.63 and $1.67 \mu \mathrm{m}$ due to $\mathrm{CH}_{4} 2 \nu_{2}$ and $2 \nu_{3}$ bands are seen. In Figure 1b, flux depression and the absorption maximum at $2.20 \mu \mathrm{m}$ of the $\nu_{2}+\nu_{3}$ band are seen.

\section{Observations, Results \& Discussion}

Observations of 2MASS 1146+22 (L3), 1507-16 (L5), and 0920+35 (L6.5) were made at Subaru in March 2001 using IRCS Grism mode. Methane absorption in $2 \mathrm{M} 0920+35$ was detected at $\mathrm{H}$ and $\mathrm{K}$ bands as described in the Figure caption.

This implies that detectable methane absorption in the $\mathrm{H}$ and $\mathrm{K}$ bands, usually considered the signature of a $\mathrm{T}$ dwarf, can be present in objects classified optically as late $\mathrm{L}$.

The presence of methane in $\mathrm{L}$ dwarfs is consistent with the prediction by the unified model of Tsuji (2002).

\section{References}

Geballe, T. et al. 1996, ApJ, 467,L101

Geballe, T. et al. 2002, ApJ, 564,466

Marley, M. et al. 2002, ApJ, 568,335

Nakajima, T., Tsuji, T. \& Yanagisawa, K. 2001, ApJ, 561, L119

Noll, K. S. et al. 2000, ApJ, 548,908

Tsuji, T. 2002, ApJ, 575, 264 